\title{
Reflets
}

Revue ontaroise d'intervention sociale et communautaire

\section{Bibliographie des articles Pratiques à notre image - 1995 à 2006}

Volume 13, numéro 1, 2007

La violence dans tous ses états

URI : https://id.erudit.org/iderudit/016819ar

DOI : https://doi.org/10.7202/016819ar

Aller au sommaire du numéro

Éditeur(s)

Reflets : Revue ontaroise d'intervention sociale et communautaire

ISSN

1203-4576 (imprimé)

1712-8498 (numérique)

Découvrir la revue

Citer ce document

(2007). Bibliographie des articles Pratiques à notre image - 1995 à 2006.

Reflets, 13(1), 208-214. https://doi.org/10.7202/016819ar

Tous droits réservés (C) Reflets : Revue ontaroise d'intervention sociale et communautaire, 2007
Ce document est protégé par la loi sur le droit d'auteur. L'utilisation des services d'Érudit (y compris la reproduction) est assujettie à sa politique d'utilisation que vous pouvez consulter en ligne.

https://apropos.erudit.org/fr/usagers/politique-dutilisation/ 


\section{Bibliographie des articles Pratiques à notre image - 1995 à 2006}

\section{Volume 1, numéro 1}

CARRIÈRE, Richard et Donald DENNIE (1995). «L'intervention sociale en français dans la région de Sudbury ", printemps, 182-190.

GEOFFRION-LOMBARDI, Micheline avec la collaboration d'Angèle Bouchard (1995). «La pratique en milieu francophone : réflexions de deux travailleuses sociales ", printemps, 175181.

POIRIER, Alain (1995). «Une exploration de modèles pour la coordination des services sociaux aux enfants et adolescents francophones ", printemps, 191-200.

TURCOTTE, Marie A. (1995). «Les services intégrés pour les enfants du Nord : une pratique socio-communautaire à notre image ", printemps, 166-174.

\section{Volume 1, numéro 2}

LABRÈCHE, Carole (1995). «Une Alliance avec la vie », automne, 166-170.

NARBONNE-FORTIN, Claire, PHILBIN WILKINSON, Hélène, LAUZON, René et Louise LAROCQUE (1995). "Politiques municipales de gestion de l'alcool : stratégie pour diminuer les problèmes reliés à la consommation risquée ", automne, 195-210.

POMERLEAU, Johanne et Manon LEMONDE (1995). «Réseau d'entraide : programme de formation personnelle auprès des femmes aînées ", automne, 171-183.

RAIL, Geneviève et Hélène DALLAIRE (1995). " "Dialogue santé " une consultation historique auprès des communautés francophones de l'Ontario ", automne, 184-194.

\section{Volume 2, numéro 1}

BOUDREAU, Francine (1996). "L'intervention contre la violence conjugale à Sudbury : aperçu et réflexions ", printemps, 98-108.

DUBORD, Léo (1996). «Transiger quotidiennement avec des phénomènes de violence : risque personnel pas toujours calculé », printemps, 134-145.

GODIN, Colette et Ghislaine SIROIS (1996). « La violence faite aux femmes : analyse de la situation en Ontario français ", printemps, 116-133. 
GRANDMONT, Thérèse (1996). «La violence démasquée », printemps, 146-154.

OUIMETTE, Johanne (1996). "Coordination : outil des années 2000 », printemps, 155-164.

PHARAND, Gaëtane (1996). «À l'aide des femmes francophones qui luttent contre les agressions à caractère sexuel ", printemps, 109-115.

\section{Volume 2, numéro 2}

LADOUCEUR, Linda (1996). "Dynamique de l'aide informelle auprès des personnes âgées ", automne, 100-108.

FAUTEUX, Andrée (1996). "Rendez-vous “ Santé globale ” ", automne, 109-127.

GRAVELLE, François, PAGEOT, Jean Claude et Julie DENIS-MÉNARD (1996). «La qualité de vie chez les personnes âgées fréquentant un centre de jour francophone de la région d'Ottawa ", automne, 118-127.

LEMONDE, Manon et Johanne POMERLEAU (1996). «Restructuration des soins de santé :impact sur les personnes âgées en Ontario ", automne, 128-135.

\section{Volume 3, numéro 1}

BAGAOUI, Rachid (1997). «Réponses des associations communautaires de la région de Sudbury à la crise de l'État providence ", printemps, 102-112.

GIGUÈRE, Carole (1997). "Coupures dans les services sociaux : impact sur les femmes victimes de violence et leurs enfants ", printemps, 113-119.

REITSMA-STREET, Marge,VAN DE SANDE, Adje et François BOUDREAU (1997). « Bilan des stratégies pour combattre la pauvreté chez les enfants et les adolescents à Sudbury ", printemps, $120-135$.

\section{Volume 3, numéro 2}

BÉLANGER, Lucie et Danielle FOURNIER (1997). «Économie sociale et solidaire ", automne, 144-155.

BOUCHARD, Lyne (1997). "Affirmation politique des femmes au sein du milieu associatif", automne, $187-200$.

BOUCHARD, Lyne (1997). "Principes féministes en milieu coopératif », automne, 156-165.

BOUCLIN, Marie (1997). "Femmes et abus de pouvoir dans l'Église ", automne, 214-227.

GUINDON, Geneviève (1997). "Féminisme des années 1990 : opinions de femmes de moins de trente ans ", automne, 201-213.

LAROCQUE, Sylvie et Christine CAVEEN (1997). "Sages-femmes : un véritable partenariat ", automne, 228-239.

PENWILL, Kathryn, PHARAND, Gaëtane, SIROIS, Ghislaine et Louise TOON (1997). «Action politique à la mode de chez nous ", automne, 166-186. 


\section{Volume 4, numéro 1}

DIALLO, Lamine et Ginette LAFRENIÈRE (1998). «Stratégies pour une redéfinition de l'espace francophone à Sudbury ", printemps, 140-150.

PARROT, Claire (1998). "Le défi des pratiques d'intervention interculturelle dans le champ de la santé sexuelle ", printemps, 128-139.

SIROIS, Ghislaine (1998). «Les femmes francophones dans le milieu ontarien de la violence faite aux femmes ", printemps, 151-163.

VAN DE SANDE,Adje et Gilles RENAULT (1998). «L'intégration des concepts autochtones dans le curriculum du travail social ", printemps, 164-150.

\section{Volume 4, numéro 2}

BRISEBOIS, Jean-Roger (1998). "Intervenir auprès des personnes ayant une perte auditive ", automne, 151-154.

GERVAIS-GUY, Louise, LAFOND, Jean et Denise BELLEHUMEUR (1998). « Nouveaux modes de services aux apprenantes et aux apprenants ayant des besoins particuliers au Collège Boréal ", automne, 123-131.

LAMBERT, Lynn (1998). « Ne pas savoir : parlons du mauvais traitement contre les femmes ayant un handicap ", automne, 132-143.

NOLET, Lise (1998). "La sclérose en plaques, ses effets et les besoins spécifiques de ceux qu'elle afflige ", automne, 144-150.

PERRON-ROACH, Danielle (1998). « L'approche institutionnelle répond-t-elle aux besoins de sa clientèle? ", automne, 106-114.

TOTH, Ann (1998). "La communication : le pont entre les Sourds et les entendants ", automne, 115-122.

\section{Volume 5, numéro 1}

BÉLANGER, Jean-Marc (1999). «GEODE (Grassroots Economic Opportunity Development and Evaluation) ", printemps, 145-149.

CHURCH, Kathryn (1999). "L'économie en tant que levier de la communauté. Les survivants psychiatriques en Ontario ", printemps, 123-144.

HAAPANEN, Paula (1999). «Le développement économique communautaire :les défis des femmes du Nord-Ouest ", printemps, 116-122.

\section{Volume 5, numéro 2}

CENTRES COMMUNAUTAIRES DE SANTÉ DE L'ESTRIE, Hamilton-Wentworth-Niagara, Sudbury,Témiskaming, Toronto (1999). « Les centres de santé communautaires :la porte ouverte à la santé des francophones ", automne, 164-178. 
CATY, Suzanne, MICHEL, Isabelle, PONG, Raymond et Dianne STEWART (1999). "Qui sont les étudiantes du programme de formation francophone d'infirmières praticiennes ", automne, 240-247.

COUTURE, Robert T. (1999). «Un centre communautaire pour la vie : un concept de “ santé de la population "pour l'an 2000 ", automne, 179-193.

DEROCHE, Francine et Anne SIMARD (1999). «La formation à distance : un moyen novateur pour promouvoir la santé ", automne, 214-225.

DEROCHE, Francine, SIMARD, Anne, DI RUGGIERO, Erica et Johanne LEVESQUE (1999). "Profil des ressources des communautés francophones en milieu minoritaire ", automne, 203213.

DORSCHNER, Danielle (1999). «Pénurie infirmière : mythe ou réalité? », automne, 235-239.

GAGNÉ, Hélène (1999). "La promotion de la santé : des pas de géants au-delà de l'an 2000 ", automne, 194-202.

LALONDE, Jocelyne (1999). "Le Réseau des services de santé en français de l'Est (RSSFE) de l'Ontario : relever les défis qui découlent de la restructuration du système de santé ", automne, 158-163.

LAROCQUE, Sylvie et Suzanne CATY (1999). «Les décisions de recherche d'emploi des nouvelles bachelières en sciences infirmières ", automne, 226-234.

MÉLANSON, Nathalie (1999). "L'évolution de la profession de sage-femme ", automne, $248-255$.

\section{Volume 6, numéro 1}

BOUDREAU, François et Herbert NABIGON (2000). "Spiritualité, guérison et autonomie gouvernementale dans le contexte politique Ojibwas ", printemps, 108-127.

DUPONT, Diane, CULLIGAN, Denyse et Gaëtane PHARAND (2000). « Un travail de défrichage : le développement des services en matière de violence faite aux femmes dans le Nord de l'Ontario ", printemps, 128-140.

GOUAULT, Michel (2000). "La magie des lettres : réflexions sur 15 années de pratique en alphabétisation en vue de l'insertion sociale des apprenantes adultes ", printemps, 154-179.

STERLIN, Carlo et François DUTHEUIL (2000). "La pratique en contexte interculturel», printemps, 141-153.

ST-DENIS, Yvonne, POPIEA, Elena et Ginette COUTU-WAKULCZYK (2000). «La famillecliente en services de santé : une approche systémique ", printemps, 180-190.

\section{Volume 6, numéro 2}

CHARPENTIER, Alain et Marie Nathalie MOREAU (2000). "D’une loi à l'autre : le système de justice pénale pour les jeunes en Ontario ", automne, 168-181.

SNOW, Claude (2000). "La participation populaire engagée et organisée : une pratique sociale à l'image des Acadiens ", automne, 182-200. 


\section{Volume 7, numéro 1}

BEAUVOLSK, Michel-André (2001). «Intervention auprès d'une adolescente dépressive en milieu scolaire ", printemps, 152-160.

BEAUVOLSK, Michel-André et Jean-Marc BÉLANGER (2001). «Pratiques quotidiennes des intervenantes et des intervenants dans la santé mentale auprès des enfants ", printemps, 114128.

PIERRE (2001). «Le combat de Catherine ", printemps, 161-164.

MUNROE,Viviane et Nicole BRUNETTE (2001). «L'épuisement professionnel (burn-out) : un problème réel ", printemps, 165-192.

VINETTE, Sophie (2001). "Image corporelle et minceur : à la poursuite d'un idéal élusif ", printemps, 129-151.

\section{Volume 7, numéro 2}

BEAUVOLSK, Michel-André (2001). "Le travail social auprès des familles ", automne, 92-103.

CARRIÈRE, Richard (2001). "Le travail social auprès des personnes âgées en Ontario ", automne, 104-117.

GASKIN, Sylvie et Michelle SPENCE (2001). «Travailleuse sociale à l'hôpital : une partenaire dans la prestation des soins de santé ", automne, 118-131.

VAN DE SANDE,Adje et Gilles RENAULT (2001). « Le travail social auprès des enfants ", automne, 76-91.

\section{Volume 8, numéro 1}

BROUILLETTE, Carl, L'ABBÉ, Bernard et Manon TREMBLAY (2002). «Équipes communautaires de traitement intensif : description et expérience des équipes parrainées par l'Hôpital Montfort ", printemps, 114-124.

CASIMIRO, Lynn et Lucie BROSSEAU (2002). "La collaboration Cochrane : un outil de travail et de recherche pour les spécialistes de la réadaptation ", printemps, 151-155.

CASIMIRO, Lynn, FORTIN, Murielle, MOISAN, France et Louis E. TREMBLAY (2002). «Le recensement des outils de travail disponibles en français pour les professionnels œuvrant dans le domaine de la santé : un rapport de la méthodologie utilisée par les physiothérapeutes ", printemps, 125-150.

GAUDET, Pierre (2002). "Le centre national de formation en santé ", printemps, 106-113.

SAVARD, Jacinthe, DUBOULOZ, Claire-Jehanne et Dawn BURNETT (2002). "Une ère de changement pour la formation en réadaptation ", printemps, 95-105.

TREMBLAY, Louis E. et François TREMBLAY (2002). "La réadaptation communautaire, une nécessité sous-exploitée dans les milieux francophones de l'Ontario ", printemps, 74-94. 


\section{Volume 9, numéro 1}

CODERRE, Cécile et Joanne HART (2003). « Pratiques d'intervention féministe auprès des femmes survivantes d'agression à caractère sexuel : le contexte franco-ontarien ", printemps, 186-210.

MICHAUD, Jacinthe (2003). "Pratiques féministes, éducation populaire et conscience identitaire, 180-185.

\section{Volume 9, numéro 2}

BOISSONNEAULT, Julie (2003). "Représentations des TIC en milieu professionnel : réflexions sur le changement ", automne, 207-221.

HOUlE, Pascale et Magnolia SOUTYRINE (2003). "Les Ailes francophones: un pas vers l'autonomie des femmes ", automne, 199-206.

LÉGÈRE, Isabelle (2003). "Enjeux de la nouvelle économie sociale dans la Péninsule acadienne », automne, 188-198.

OUELLET, Francine et Gilles FOR GET (2003). « Pères en mouvement / Pratiques en changement : une formation pour favoriser le transfert des connaissances ", automne, 222-240.

RENAULT, Gilles (2003). "Le Consortium national de formation en santé ", automne, 241-252.

\section{Volume 10}

BÉLANGER, Jean-Marc (2004). «Les jeunes de la rue de 16 à 17 ans à Sudbury : «Tombés entre les mailles du filet ", 106-111.

RINGUET, Caroline (2004). «La sensibilisation « au quotidien » : atelier au sujet de l'homosexualité dans une école secondaire ", 100-105.

ST-AMAND, Nérée avec la collaboration de Manon Arsenault (2004) «Le Théâtre des Lueurs : une lueur d'espoir?", 112-115.

\section{Volume 11}

BYRNE, Beverly Ann et Raymond LEMAY (2005). « Parole aux jeunes : les jeunes nous disent ce qu'il faut pour assurer le succès du placement résidentiel ", 196-213.

DESBIENS, Francine (2005). « Le démarrage d'un programme national de recherche sur la santé des communautés francophones minoritaires - une initiative du Consortium national de formation en santé ", 187-195.

GARCEAU, Marie-Luce (2005). "Réflexions sur le développement des services en matière de violence ", 148-164.

LEGAULT, Marie-Josée (2005). "Les Francophones d'Ottawa : une communauté diverse ", vol. 11,165-177.

PLANTE, Nathalie, BASSOLÉ, Angèle, HAMBOYAN, Hoori, KÉRISIT, Michèle et Marta YOUNG (2005). "L'impact du conflit armé sur l'intégration des femmes immigrantes ", vol. $11,178-186$. 


\section{Volume 12}

BRUNET, Lucie (2006). « Spiritualité et intervention sociale », vol. 12, 181-189.

DUPONT, Diane (2006). "Sortir la spiritualité du placard ", vol. 12, 168-180.

GAGNÉ, Pauline (2006). «Spiritualité et intervention : convergence et défis », vol. 12, 190-196. MALTAIS, Ronald (2006). « Expérience spirituelle », vol. 12, 197-200.

THIBODEAU, Lison (2006). « La communauté de l'Arche », vol. 12, 201-205. 